Beitrag von Roman Opilowski dar, der eine linguistische Analyse von Bild und Text anhand von Werbung vorlegt. Eine andere fruchtbare Forschungsrichtung stellt Waldemar Czachur vor, der nach kulturspezifischen Ausprägungen von diskursiven Strategien am Beispiel Polens und Deutschlands zum Gaskonflikt von 2009 fragt. Czachur ersetzt quasi den Dispositivbegriff mit Kultur, ohne das zu begründen.

Der Band bietet einen spannenden Einblick in den Komplex Medien, Diskurs, Dispositiv, Macht. Die Herausforderung für weitere Denkbewegungen besteht zum einen in der Erweiterung des Materials um Bilder und Klang, zum anderen aber in der weiteren Reflektion über das Verhältnis von Dispositiv und Diskurs. Die Frage, die die diskursanalytisch arbeitenden Forscher und Forscherinnen immer noch umtreibt, ist die nach dem Verhältnis von Diskursivität und dem „anderen“ (Reiner Keller), bzw. nach einer Differenz zwischen diskursiven und nicht-diskursiven Praxen. In einer radikalen Zuspitzung, die Achim Landwehr vorschlägt, gib es kein Außerhalb des Diskurses, wobei er damit Sprachliches und Nichtsprachliches einschließt. ${ }^{1}$ Zugleich aber scheint gerade, wenn es um Medien als Untersuchungsgegenstand geht, die Vorstellung des Dispositivs fruchtbar, eben weil hierdurch sowohl die Produktivität der Macht beschrieben werden als auch die bisherige Bipolarität von „Medienmacht“ aufgelöst werden kann. Des Weiteren bedeutet die Historisierung des Dispositivs eine Möglichkeit, die Transformation von den Produktionen der Ordnung des Diskurses zu untersuchen und so die Veränderungen der Formationsregeln genauer zu beschreiben.

Inge Marszolek

\section{Jörg Hagenah / Heiner Meulemann (Hrsg.) Mediatisierung der Gesellschaft?}

Berlin: LIT, 2012. - 314 S.

ISBN 978-3-643-11594-2

Sammelbandbesprechungen sind immer ungerecht. Die größte Ungerechtigkeit dabei ist die Verkürzung theoretischer Argumente oder empirischer Befunde auf wenige Zeilen. Der von Natur aus ungerechte Sammelbandrezensent muss zwischen zwei Übeln wählen: Entweder er reduziert die Komplexität aller Beiträge auf wenige Worte, um entgegen besseren Wissens es doch allen Recht machen zu wollen, oder er adelt ausgewählte Beiträge durch ausführliche Besprechung, während der Rest zur Randnotiz gerät oder gleich ganz ignoriert wird. Der Vollständigkeit verpflichtete Sammelbandrezensenten werden es zudem nicht versäumen, an irgendeiner Stelle die Leistung der Herausgeber arg verkürzt zu würdigen.

Heiner Meulemann und Jörg Hagenah von der Universität zu Köln haben im November 2010 im Rahmen ihres DFG-Projekts „Neue Rundfunkangebote und alte Gewohnheiten" eine Tagung mit dem Titel „Mediatisierung der Gesellschaft? Mediensoziologische Forschung in vergleichender Perspektive" ausgerichtet. Ausgewählte Beiträge der Tagung sind im Sammelband dokumentiert. Nach einem Vorwort der Herausgeber leitet ein Beitrag von Friedrich Krotz das Buch ein. Nichts liegt natürlich näher, als Krotz die theoretische Einführung zu überlassen - zumal er eine von drei Keynotes auf der Tagung gehalten hat. Nur wer überhaupt nicht mit dem Mediatisierungsbegriff von Krotz vertraut ist, dürfte diese langatmige deutsche Fassung seines pointiert geschriebenen Kurzaufsatzes "The meta-process of 'mediatization' as a conceptual frame“ (2007) mit Gewinn lesen. Mediatisierung, so Krotz, sei als ein Metaprozess zu verstehen, ähnlich wie die Globalisierung oder Individualisierung. Dieser Metaprozess kennzeichnet den fortwährenden „Wandel von Kommunikation“ (S. 37) in der Gesellschaft.

Der zweite, rein theoretische Beitrag über Netzöffentlichkeit stammt von Norbert Schneider, der im Internet trotz zunehmender Reichweite kein Leitmedium erkennen mag, das einer Regulierung vorherrschender Meinungsmacht bedarf. Vielmehr sorgen den ehemaligen Medienaufseher - lange vor Bekanntwerden der NSA-Affäre - die „Kontrolleure, die niemand kontrolliert" (S. 52).

Beim Lesen fällt auf, dass die im Band dokumentierten Studien, denen fast allen entweder MA- oder andere Sekundärdaten zugrunde liegen, meist nur ein paar pflichtschuldige Referenzen zum Mediatisierungsdiskurs enthalten. Überrascht hat mich, wie leichtfertig die Autoren immer wieder in einem Satz über Mediatisierung und Medialisierung schreiben. Für Marco Dohle und Gerhard Vowe sind dies offenbar nur „Begriffsvarianten“ (S. 277), was der hart geführten begriffstheoretischen Debatte sicherlich nicht gerecht wird und eingefleischten

1 Landwehr, Achim (2010): Abschließende Betrachtungen: Kreuzungen, Wiederholungen, Irritationen, Konflikte, in: Ders. (Hrsg.), Diskursiver Wandel. Wiesbaden, S. 383. 
Medialisierungsforschern die Haare zu Berge stehen lassen wird (vgl. z. B. Meyen 2009).

Die Herausgeber argumentieren, dass der Begriff der Mediatisierung mit der These verbunden sei, "dass sich der Einfluss massenhaft genutzter Medien auf den Lebensalltag der Bevölkerung in den letzten Jahren verstärkt hat" (S. 7). Ich fand es beim Lesen schade, dass in keinem der Beiträge diese These im Licht der empirischen Ergebnisse diskutiert worden ist. So bleibt der Eindruck hängen, dass der Band eine lose Ansammlung von Sekundärstudien ohne Einbettung in einen theoretischen Gesamtzusammenhang ist.

Heiner Meulemann, Beate Apolinarski und David Gilles zeichnen mithilfe der Daten der Media-Analyse für elektronische Medien nach, dass im Alter die Nutzung von Internet-Anwendungen (wie z. B. Suchmaschinen, E-Mail, Online-Einkäufe usw.) nachlässt. Von diesen Anwendungen dürfte aktuell keine Mediatisierung des Alters zu erwarten sein. Sabine Hake beschreibt anhand der MA-Daten die Kinogängerschaft zwischen 1976 und 2006. Wie bei allen Regressionsanalysen im Sammelband, die auf MA-Daten beruhen, zeigt sich auch bei Hake der hohe Preis, den man zahlen muss, wenn man zu große Stichproben hat: 99,9 Prozent aller Effekte sind mit drei Sternchen versehen; jeder noch so kleine Effekt ist signifikant. PowerAnalysen wären hier wohl angebracht gewesen, um substanzielle Effekte identifizieren zu können.

Michael Schenk und Cornelia Jers zeigen mithilfe der Datenbasis der ARD/ZDF-Langzeitstudie Massenkommunikation, dass sich die Orientierungs-, Informations- und Eskapismusfunktion von Tageszeitung, Fernsehen und Radio seit den 1970 er Jahren kaum verändert haben. Die Analyse von Hans-Jörg Stiehler veranschaulicht, dass Ostdeutsche in der Zeit zwischen 1995 und 2005 konstant intensiver privates Fernsehen genutzt und weniger häufig überregionale Tageszeitungen gelesen haben als Westdeutsche. Der von Birgit Stark, Nicole Gonser und Thorsten Naab durchgeführte Vergleich deutscher und österreichischer MA-Daten macht deutlich, dass die Reichweiten der Print-Leitmedien in beiden Ländern zwischen 1997 und 2007 nahezu gleich geblieben sind und dass sich die Leserschaft in dieser Zeit kaum verändert hat. Die auf Basis der MA-Intermedia durchgeführte Publikumsanalyse von Damir Babić und Jörg Hagenah belegt, dass die Ausdifferenzierung des Marktes privater Fernsehanbieter in Deutschland - abgesehen von einem Periodeneffekt zwischen 2001 und 2004 sich zwischen 1998 und 2009 nicht negativ auf die Nutzungswahrscheinlichkeit von RTL, Sat. 1 und ProSieben ausgewirkt hat.

Insbesondere die empirischen Stabilitätsbefunde hätten meiner Meinung nach dringend der Einordnung bedurft: Welche theoretischen Konsequenzen sind daraus zu ziehen, dass die Nutzungspraxis einzelner Medien zeitstabil ist? Entspricht dieses Ergebnis dem, was Mediatisierungsforscher neuerdings als Beharrung bezeichnen? Hätte der Untersuchungszeitraum einfach noch länger sein müssen? Oder sind die Indikatoren der MA-Daten gar ungeeignet, um den von Krotz beschriebenen Wandel von Kommunikation empirisch nachzuvollziehen? Es ist vielleicht der Entstehungsgeschichte des DFG-Projekts geschuldet, dass der Sammelband vor allem Ausdruck der vielfältigen Anwendbarkeit von MA-Daten und anderer Längsschnittdaten ist und eine abschließende theoretische Einordnung auf der Strecke blieb. Mit dem DFG-Projekt ist zunächst einmal ein methodisches Ziel verfolgt worden: die Nutzbarmachung einer kommerziellen Datenquelle für wissenschaftliche Sekundärforschung. Dieses Ziel haben die Antragsteller vorbildlich gelöst, wie ich finde. Möglicherweise hätte der Sammelband überzeugender auf den Leser gewirkt, wenn mehr methodische Beiträge wie die von Bertram Scheufele oder Jörg Hagenah und Dominik Becker den Band geschmückt hätten. Die rein methodischen Beiträge der drei Autoren sind für all diejenigen wertvoll, die sich über Prinzipien und Techniken der Datenfusion informieren möchten, um ein Verständnis für die Logik und Struktur der MA-Daten zu entwickeln.

Auch wenn es von außen nie so scheint, jede Sekundäranalyse ist - wie die Erhebung von Primärdaten - auch ein kreativer Akt, der ohne Fantasie nicht möglich gewesen wäre. Wer sich informieren möchte, auf welche verschiedene Weise die MA-Daten sekundäranalytisch einsetzbar sind, wird in vielen Beiträgen des Sammelbands fündig. Auch wenn der Sammelband an sich mich nicht überzeugt hat, die Studien darin habe ich mit Gewinn gelesen.

Jens Vogelgesang

\section{Literatur}

Krotz, Friedrich (2007): The meta-process of ,mediatization' as a conceptual frame. Global Media and Communication, 3(3), S. 256-260.

Meyen, Michael (2009): Medialisierung. In: Medien \& Kommunikationswissenschaft, 57(1), S. 23-38. 